\title{
The last fraction of a fractional conjecture
}

\author{
František Kardoš* $\quad$ Daniel Král’† Jean-Sébastien Sereni ${ }^{\ddagger}$
}

\begin{abstract}
Reed conjectured that for every $\varepsilon>0$ and every integer $\Delta$, there exists $g$ such that the fractional total chromatic number of every graph with maximum degree $\Delta$ and girth at least $g$ is at most $\Delta+1+\varepsilon$. The conjecture was proven to be true when $\Delta=3$ or $\Delta$ is even. We settle the conjecture by proving it for the remaining cases.
\end{abstract}

\section{Introduction}

Fractional graph theory has led to many elegant and deep results in the last three decades, broadening the range of applications of graph theory and providing partial results and insights to many hard problems. In this paper, we are interested in fractional total colorings of graphs. Total colorings form an extensively studied topic - see the monograph by Yap [11 — with the Total Coloring Conjecture of Behzad [1] and Vizing [10] being one of its grails.

Conjecture 1 (Total Coloring Conjecture). The total chromatic number of every graph with maximum degree $\Delta$ is at most $\Delta+2$.

*Institute of Mathematics, Faculty of Science, University of Pavol Jozef Šafárik, Jesenná 5, 04154 Košice, Slovakia, and Institute for Theoretical Computer Science, Faculty of Mathematics and Physics, Charles University, Prague, Czech Republic. Partially supported by the Slovak Science and Technology Assistance Agency under the contract No APVV-0007-07. E-mail: frantisek.kardos@upjs.sk.

†Institute for Theoretical Computer Science, Faculty of Mathematics and Physics, Charles University, Malostranské náměstí 25, 11800 Prague, Czech Republic. E-mail: kral@kam.mff.cuni.cz. The Institute for Theoretical Computer Science (ITI) is supported by Ministry of Education of the Czech Republic as project 1M0545. This research has been supported by grant GACR 201/09/0197.

${ }^{\ddagger}$ CNRS (LIAFA, Université Denis Diderot), Paris, France, and Department of Applied Mathematics (KAM), Faculty of Mathematics and Physics, Charles University, Prague, Czech Republic. E-mail: sereni@kam.mff.cuni.cz. 
The most important partial result toward the Total Coloring conjecture to date is the following theorem proved by Molloy and Reed [7] in 1998.

Theorem 2. The total chromatic number of every graph with maximum degree $\Delta$ is at most $\Delta+10^{26}$.

As is often the case, the fractional analogue of Conjecture 1 turns out to be easier to approach. Kilakos and Reed [5] proved in 1993 the following fractional analogue of the Total Coloring Conjecture.

Theorem 3. The fractional total coloring number of every graph with maximum degree $\Delta$ is at most $\Delta+2$.

The graphs achieving the bound given in Theorem 3 have been identified by Ito, Kennedy and Reed [2]: they are the complete graphs of even order and the complete bipartite graphs with equal part sizes. Inspired by these results, Reed [8] conjectured the following.

Conjecture 4. For every $\varepsilon>0$ and every integer $\Delta$, there exists $g$ such that the fractional total chromatic number of every graph with maximum degree $\Delta$ and girth at least $g$ is at most $\Delta+1+\varepsilon$.

Conjecture 4 was proven to be true when $\Delta \in\{3\} \cup\{4,6,8,10, \ldots\}$ by Kaiser, Král' and King [3] in the following stronger form.

Theorem 5. Let $\Delta \in\{3\} \cup\{4,6,8,10, \ldots\}$. There exists $g$ such that the fractional total chromatic number of every graph with maximum degree $\Delta$ and girth at least $g$ is $\Delta+1$.

The purpose of our work is to settle Conjecture 4 by proving that it also holds for odd values of $\Delta$. Our main theorem reads as follows.

Theorem 6. For every $\varepsilon>0$ and every odd integer $\Delta \geq 5$, there exists an integer $g$ such that for the fractional total chromatic number of every graph with maximum degree $\Delta$ and girth at least $g$ is at most $\Delta+1+\varepsilon$.

The approach we use also yields a proof for the case where $\Delta$ is even, and thus a full proof of Conjecture 4. However, we restrict ourselves to the case of odd $\Delta \geq 5$, since a stronger result, Theorem [5, has been established [3]. Kaiser et al. [3] also conjectured that the statement of Theorem 5 also holds for odd values of $\Delta$.

Conjecture 7. Let $\Delta \geq 5$ be an odd integer. There exists $g$ such that the fractional total chromatic number of every graph with maximum degree $\Delta$ and girth at least $g$ is $\Delta+1$.

However, we were not able to settle this stronger conjecture. 


\section{Definitions and notation}

Let us start by defining the relevant concepts. For $X \subseteq \mathbb{R}$, we define $\mu(X)$ to be the Lebesgue measure of $X$. If $G$ is a graph, then $V(G), E(G)$ and $\Delta(G)$ are its vertex-set, edge-set and maximum degree, respectively. The total graph $\mathcal{T}(G)$ of $G$ is the graph with vertex-set $V(G) \cup E(G)$, where two vertices are adjacent if and only if the corresponding elements of $G$ are adjacent or incident. In other words, $x y \in E(\mathcal{T}(G))$ if and only if

- $x, y \in V(G)$ and $x y \in E(G)$, or

- $x, y \in E(G)$ and $x$ and $y$ share a vertex in $G$, or

- $x \in V(G), y \in E(G)$ and $y$ is incident to $x$ in $G$.

A total independent set of $G$ is an independent set of $\mathcal{T}(G)$. Let $\Phi(G)$ be the set of all total independent sets of $G$.

Consider a function $w: \Phi(G) \rightarrow[0,1]$ and let $x \in V(\mathcal{T}(G))$. We define $w[x]$ to be the sum of $w(I)$ over all $I \in \Phi(G)$ containing $x$. The mapping $w$ is a fractional $k$-total coloring of $G$ if and only if

- $\sum_{I \in \Phi(G)} w(I) \leq k ;$ and

- $w[x] \geq 1$ for every $x \in V(\mathcal{T}(G))$.

Observe that $G$ has a fractional $k$-total coloring if and only if there exists a function $c: V(\mathcal{T}(G)) \rightarrow 2^{[0, k]}$ such that

- $\mu(c(x)) \geq 1$ for every $x \in V(\mathcal{T}(G))$; and

- $c(x) \cap c(y)=\emptyset$ for every edge $x y \in E(\mathcal{T}(G))$.

Notice that the second condition is the same as to require that $\mu(c(X) \cap$ $c(Y))=0$ whenever $x$ and $y$ are adjacent in $\mathcal{T}(G)$, since we consider only finite graphs. The fractional total chromatic number of $G$ is the infimum of all positive real numbers $k$ for which $G$ has a fractional $k$-total coloring. As is well-known, the fractional total chromatic number of a finite graph is always a rational number and the infimum is actually a minimum.

A mapping $w: \Phi(G) \rightarrow[0,1]$ such that

- $\sum_{I \in \Phi(G)} w(I)=1$; and

- $w[x] \geq \alpha$ for every $x \in V(\mathcal{T}(G))$ 
is a weighted $\alpha$-total coloring of $G$. Observe that every fractional $k$-total coloring yields a weighted $\frac{1}{k}$-total coloring $w$ such that $w[x] \geq 1 / k$ for every $x \in V(\mathcal{T}(G))$. Conversely, one can derive a fractional $\frac{1}{\alpha}$-total coloring from a weighted $\alpha$-total coloring of $G$. There are many equivalent definitions of a fractional coloring of a graph, and we refer to the book by Scheinerman and Ullman [9] for further exposition about fractional colorings (and, more generally, fractional graph theory).

We now introduce some additional notation. Two functions $f, g: X \rightarrow Y$ agree on $Z \subseteq X$ if the restrictions of $f$ and $g$ to $Z$ are equal. Let $G$ be a graph and $v \in V(G)$. For a spanning subgraph $F$ of $G$, the degree of the vertex $v$ in $F$ is $\operatorname{deg}_{F}(v)$. A spanning subgraph of $G$ with maximum degree at most 2 is a sub-2-factor of $G$. An $\ell$-decomposition of $G$ is a partition of the edges of $G$ into $\lceil\ell / 2\rceil$ sub-2-factors, one of which is required to be a matching if $\ell$ is odd.

Given a connected graph $G$, an edge-cut $F$ of $G$ is a subset of edges such that the removal of $F$ disconnects $G$. Note that removing a minimal edge-cut splits $G$ into exactly two connected components. An edge-cut $F$ is cyclic if every connected component of $G-F$ contains a cycle. A graph is cyclically $k$-connected if it has more than $k$ edges and no cyclic edge-cut of size less than $k$.

We also use the following terminology from [4]. Let $H$ be a subgraph of a connected graph $G$. A path $P$ of $G$ is an $H$-path if both end-vertices of $P$ belong to $H$ but no internal vertex and no edge of $P$ belongs to $H$. Given an integer $d$, the subgraph $H$ is $d$-closed if the length of every $H$-path is greater than $d$. The $d$-connector of $H$ in $G$ is the smallest $d$-closed subgraph of $G$ that contains $H$. The neighborhood $N(H)$ of $H$ is the subgraph of $G$ spanned by all the edges of $G$ with at least one end-vertex in $H$. We end this section by citing a lemma of Kaiser et al. [4, Lemma 8] about connectors.

Lemma 8. Let $d>\ell \geq 1$. Suppose that $H$ is a subgraph of $G$ with at most $\ell$ edges and no isolated vertices. If the girth of $G$ is greater than $(d+1) \ell$, then the neighborhood of the d-connector of $H$ is a forest.

\section{The cyclically $\Delta$-edge-connected case}

We find a fractional $(\Delta+1+\varepsilon)$-total coloring of a given cyclically $\Delta$-edgeconnected graph $G$ with odd maximum degree $\Delta \geq 5$ in the following way: first, we decompose $G$ into a matching and a set of sub-2-factors, we then search for suitable weighted colorings corresponding to the factors and combine them into a weighted $\frac{1}{\Delta+1+\varepsilon}$-total coloring of $G$. 
To find the decomposition, we use the following proposition [4, Proposition 1].

Proposition 9. Every cyclically $\Delta$-edge-connected graph with maximum degree $\Delta$ has a $\Delta$-decomposition.

For the next step, we need the following lemma.

Lemma 10. Fix a positive real number $\varepsilon$ and a positive integer $\Delta \geq 4$. There is an integer $g$ such that for every graph $G$ with maximum degree $\Delta$ and girth at least $g$, and for every sub-2-factor $F$ of $G$ such that $\Delta(G-F) \leq \Delta-2$, there exists a weighted total coloring $w$ of $G$ such that

- $\forall v \in V(G), \quad w[v] \geq \frac{1}{\Delta+\varepsilon} ;$ and

- $\forall f \in E(F), \quad w[f] \geq \frac{\Delta-1}{2(\Delta+\varepsilon)}$.

Actually, Lemma 10] is implicit in [3]. More precisely, the following is proven.

Lemma 11 ([3, Lemma 18]). Let $\Delta \geq 4$. For every $\varepsilon_{0}>0$, there exist $g \in \mathbb{N}$ and $\alpha, \beta, \gamma \in \mathbb{R}^{+}$satisfying $(\Delta-2) \alpha+\beta+2 \gamma=1, \alpha<\varepsilon_{0}$ such that for every graph with maximum degree $\Delta$ and girth at least $g$, and every 2 -factor $F$ of $G$, there exists a function $w: \Phi(G) \rightarrow[0,1]$ such that for every $x \in V(G) \cup E(G)$

$$
w[x]= \begin{cases}\beta & \text { if } x \in V(G), \\ \gamma & \text { if } x \in E(F), \\ \alpha & \text { otherwise. }\end{cases}
$$

Actually, in Lemma 18, the parameter $\alpha$ is upper-bounded by $4 / 3 \ell$, with $\ell$ depending only on the girth of $G$; hence, it suffices to take $g$ sufficiently large to obtain the inequality stated in Lemma 11.

As a crucial observation, Kaiser et al. [3, Proposition 19] proved that in Lemma 11 (Lemma 18 in [3]), the parameter $\beta$ can be chosen to be any value from the interval $\left(\varepsilon_{0}, Q(1)\right)$ for a function $Q$ defined in 3 . A more careful upper bound of one of the parameters in the proof of their Proposition 19 allows us to ensure that $\beta$ can be chosen to be $\frac{1}{\Delta+\varepsilon}$ : it suffices to prove that $Q(1) \geq \frac{1}{\Delta}$. For $\Delta \in\{4,5,6\}$ it can be checked directly, and for $\Delta \geq 7$ we have

$$
Q(1)=\frac{1-F(1)^{2}}{2} \quad \text { where } \quad F(1) \leq 1-\frac{1}{\Delta-2} .
$$

Since it holds that

$$
1-\frac{1}{\Delta-2} \leq \sqrt{1-\frac{2}{\Delta}}
$$


the inequality $Q(1) \geq \frac{1}{\Delta}$ follows.

We are now ready to explain how to derive Lemma 10 from Lemma 11.

Proof of Lemma 10. Set $\varepsilon_{0}:=\frac{\varepsilon}{(\Delta-2)(\Delta+\varepsilon)}$, and let $g, \alpha, \beta, \gamma$ be the constants given by Lemma 11. Let $G$ be a graph with maximum degree $\Delta$ and girth at least $g$, and $F$ be a sub-2-factor of $G$. We first build an auxiliary graph $\widehat{G}$ as follows.

Set $k:=\sum_{v \in V(G)}\left(2-\operatorname{deg}_{F}(v)\right)$. We view $G$ as the subgraph of the multigraph $G^{\prime}$ obtained by adding to $G$ a new vertex $v_{0}$ and, for each $v \in V(G)$, adding $2-\operatorname{deg}_{F}(v)$ edges between $v_{0}$ and $v$. Thus, $\operatorname{deg}_{G^{\prime}}\left(v_{0}\right)=k$ and every other vertex of $G^{\prime}$ has degree at most $\Delta$, since $\Delta(G-F) \leq \Delta-2$. Let $H$ be a $k$-regular graph of girth at least $g$. The existence of such a graph is well-known, consult, e.g., the book by Lovász [6, Solution of Problem 10.12]. Replace every vertex $x$ of $H$ by a copy $G_{x}$ of $G$ and identify the $k$ edges incident with $x$ in $H$ with the $k$ edges of $G^{\prime}$ incident with $v_{0}$. Let $\widehat{G}$ be the obtained graph. For $x \in V(H)$, let $F_{x}$ be the set of edges of $G_{x}$ corresponding to the edges of $G$ that are in $F$. Define $\widehat{F}$ to be the union of $\bigcup_{x \in V(H)} F_{x}$ and the edges corresponding to those of $H$; thus $\widehat{F}$ is a 2-factor of $\widehat{G}$.

By the construction, $\widehat{G}$ has maximum degree $\Delta$ and girth at least $g$. Thus, Lemma 11 ensures the existence of a weighted total coloring $w$ of $\widehat{G}$ such that $\alpha \leq \varepsilon_{0}$, the weight of the vertices is $\beta=\frac{1}{\Delta+\varepsilon}$ and the weight of the edges in $\widehat{F}$ is

$$
\gamma=\frac{1}{2}(1-(\Delta-2) \alpha-\beta) \geq \frac{1}{2}\left(1-\frac{\varepsilon}{\Delta+\varepsilon}-\frac{1}{\Delta+\varepsilon}\right)=\frac{\Delta-1}{2(\Delta+\varepsilon)} .
$$

This yields the conclusion since $\widehat{G}$ contains $G$ as a subgraph and $\widehat{F}$ contains $F$.

We conclude the section by proving Theorem [6 restricted to cyclically $\Delta$-edge-connected graphs of maximum degree $\Delta$.

Lemma 12. Let $\Delta$ be an odd integer and $\varepsilon$ a positive real. There exists $g$ such that the fractional total chromatic number of every cyclically $\Delta$-edgeconnected graph with maximum degree $\Delta$ and girth at least $g$ is at most $\Delta+1+\varepsilon$.

Proof. We may assume that $\Delta \geq 5$. Set $k:=\lfloor\Delta / 2\rfloor$ and $\varepsilon^{\prime}:=\varepsilon / 2$. Let $g$ be large enough so that Lemma 10 holds for the fixed values of $\Delta$ and $\varepsilon^{\prime}$.

By Proposition 9, the graph $G$ has a $\Delta$-decomposition $M, F_{1}, F_{2}, \ldots, F_{k}$ where $M$ is a matching. In particular, $\Delta\left(G-F_{i}\right) \leq \Delta-2$ for $i \in\{1, \ldots, k\}$. For every $i \in\{1,2, \ldots, k\}$, let $w_{i}^{\prime}$ be a weighted total coloring of $G$ given 
by Lemma 10 applied to $G$ and $F_{i}$ with respect to $\Delta$ and $\varepsilon^{\prime}$. Further, let $w_{0}^{\prime}$ be the weighted total coloring of $G$ that assigns 1 to the set $M$ and 0 to every other total independent set of $G$. Finally, set $w_{i}:=\frac{2 k+1}{2 k(k+1)} \cdot w_{i}^{\prime}$ for $i \in\{1,2 \ldots, k\}$ and $w_{0}:=\frac{1}{2 k+2} \cdot w_{0}^{\prime}$.

A weighted $1 /(\Delta+1+\varepsilon)$-coloring $w: \Phi(G) \rightarrow \mathbb{R}$ is defined by setting $w:=\sum_{i=0}^{k} w_{i}$. Note that $w$ is a weighted total coloring of $G$ since it is a convex combination of weighted total colorings of $G$.

It remains to show that $w[x]$ is at least $\frac{1}{\Delta+1+\varepsilon}=\frac{1}{2 k+2+\varepsilon}$ for every $x \in$ $V(\mathcal{T}(G))$. Let $v \in V(G)$. Then, $w_{i}[v] \geq \frac{1}{\Delta+\varepsilon^{\prime}}=\frac{2}{2(2 k+1)+\varepsilon}$ for each $i \in$ $\{1,2, \ldots, k\}$, and hence

$$
\begin{aligned}
w[v] & \geq \sum_{i=1}^{k} w_{i}[v] \\
& \geq k \cdot \frac{2 k+1}{2 k(k+1)} \cdot \frac{2}{2(2 k+1)+\varepsilon} \\
& =\frac{1}{(k+1) \cdot\left(2+\frac{\varepsilon}{2 k+1}\right)} \\
& >\frac{1}{2 k+2+\varepsilon} .
\end{aligned}
$$

Now, let $e \in E(G)$. If $e \in M$, then $w[e] \geq w_{0}(M)=\frac{1}{2 k+2}$. Otherwise, there exists a unique $i \in\{1,2, \ldots, k\}$ such that $e \in E\left(F_{i}\right)$. Then,

$w[e] \geq w_{i}[e] \geq \frac{2 k+1}{2 k(k+1)} \cdot \frac{2 k}{2\left(2 k+1+\varepsilon^{\prime}\right)}=\frac{1}{(k+1) \cdot\left(2+\frac{\varepsilon}{2 k+1}\right)}>\frac{1}{2 k+2+\varepsilon}$.

This concludes the proof.

\section{The general case}

We start with an auxiliary lemma regarding recoloring of trees.

Lemma 13. Fix $\varepsilon>\varepsilon^{\prime}>0$ and a positive integer $\Delta$. There exists an integer $d$ such that the following holds.

- For every tree $T$ rooted at a leaf $r$, with maximum degree $\Delta$ and depth $d$,

- for every fractional $\left(\Delta+1+\varepsilon^{\prime}\right)$-total coloring $c_{0}$ of $T$ with colors contained in $\left[0, \Delta+1+\varepsilon^{\prime}\right]$, and 


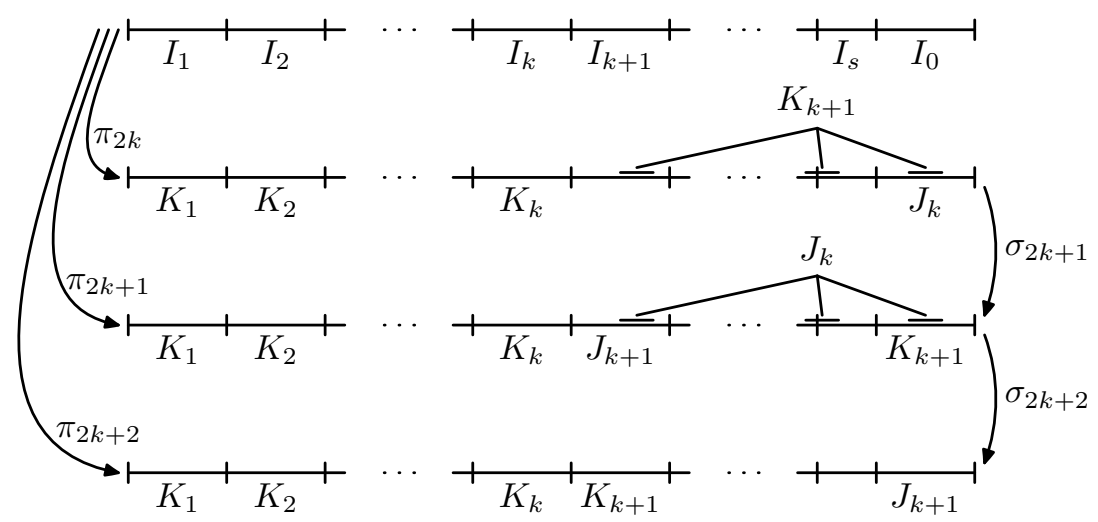

Figure 1: An illustration of the actions of the isometries $\pi_{2 k+1}$ and $\pi_{2 k}$. The colors in $K_{k+1}$ are not used on level $2 k+1$, and the colors in $J_{k+1}$ are not used on level $2 k+2$. Moreover, $\pi_{2 k}$ and $\pi$ agree on $I_{1} \cup \ldots \cup I_{k}$.

- for every pair of disjoint sets $X, Y \subset[0, \Delta+1+\varepsilon]$ each of measure 1 ,

there exists a fractional $(\Delta+1+\varepsilon)$-total coloring $c$ of $T$ such that

1. $c(r)=X$ and $c\left(r r^{\prime}\right)=Y$ where $r^{\prime}$ is the unique neighbor of $r$ in $T$, and

2. $c$ agrees with $c_{0}$ on all leaves and edges incident with leaves that are at distance $d$ from $r$.

Proof. Without loss of generality, we may assume that $T$ is $\Delta$-regular, i.e., every vertex of $T$ has degree either 1 or $\Delta$, and all leaves are at distance $d$ from $r$. Set $\delta:=\varepsilon-\varepsilon^{\prime}, s:=\left\lceil\frac{\Delta+1+\varepsilon^{\prime}}{\delta}\right\rceil$ and $d:=2 s+1$. We partition the vertices and edges of $T$ into levels regarding their distance (in the total graph of $T$ ) from $r$. More precisely, the root $r$ has level $d$, and for every $i \geq 0$, the level of a vertex at distance $i$ (in $T$ ) from $r$ is $d-i$. Thus, the leaves of $T$ distinct from $r$ have level 0 . The level of an edge that joins a vertex of level $i$ with a vertex of level $i+1$ is $i$.

We fix an isometry $\pi$ of $[0, \Delta+1+\varepsilon]$ such that $\pi\left(c_{0}(r)\right)=X$ and $\pi\left(c_{0}\left(r r^{\prime}\right)\right)=Y$. This is possible since $\mu\left(c_{0}(r)\right)=\mu\left(c_{0}\left(r r^{\prime}\right)\right)=\mu(X)=$ $\mu(Y)=1$ and $c_{0}(r) \cap c_{0}\left(r r^{\prime}\right)=\emptyset=X \cap Y$. Let $I_{1}, \ldots, I_{s}$ be a partition of $\left[0, \Delta+1+\varepsilon^{\prime}\right]$ into sets of measure $\delta$, except $I_{s}$ which may have a smaller measure; let $I_{0}=\left(\Delta+1+\varepsilon^{\prime}, \Delta+1+\varepsilon\right]$. We set $K_{k}:=\pi\left(I_{k}\right)$ for $k \in\{1, \ldots, s\}$.

Let $\pi_{0}$ be the identity mapping of $[0, \Delta+1+\varepsilon]$. We use a finite induction to define a sequence $\pi_{1}, \ldots, \pi_{2 d}$ of isometries of $[0, \Delta+1+\varepsilon]$ such that for each $k$, the isometries $\pi_{2 k}$ and $\pi$ agree on the sets $I_{1}, \ldots, I_{k}$. The definition may be better digested with a look at Figure 1 . 
Let $k \in\{0, \ldots, s-2\}$ and assume that $\pi_{2 k}$ is an isometry of $[0, \Delta+1+\varepsilon]$ such that

$$
\forall i \in\{1, \ldots, k\}, \quad \forall t \in I_{i}, \quad \pi_{2 k}(t)=\pi(t) .
$$

Let $J_{k}:=\pi_{2 k}\left(I_{0}\right)$, so $J_{0}=I_{0}$.

In the odd step, we fix an isometry $\sigma_{2 k+1}: J_{k} \rightarrow K_{k+1}$ such that the restriction of $\sigma_{2 k+1}$ to $J_{k} \cap K_{k+1}$ is the identity mapping. The isometry $\pi_{2 k+1}$ of $[0, \Delta+1+\varepsilon]$ is defined by

$$
\pi_{2 k+1}(t):= \begin{cases}\sigma_{2 k+1}\left(\pi_{2 k}(t)\right) & \text { if } t \in \pi_{2 k}^{-1}\left(J_{k}\right)=I_{0}, \\ \sigma_{2 k+1}^{-1}\left(\pi_{2 k}(t)\right) & \text { if } t \in \pi_{2 k}^{-1}\left(K_{k+1}\right), \\ \pi_{2 k}(t) & \text { otherwise. }\end{cases}
$$

For $i \in\{1, \ldots, k\}$, if $t \in I_{i}$ then $t \notin \pi_{2 k}^{-1}\left(K_{k+1}\right)$ since $\pi_{2 k}(t)=\pi(t) \in K_{i}$, and $t \notin I_{0}$ since $I_{0} \cap I_{i}=\emptyset$. Therefore,

$$
\forall i \in\{1, \ldots, k\}, \quad \forall t \in I_{i}, \quad \pi_{2 k+1}(t)=\pi_{2 k}(t)=\pi(t) .
$$

Note that $\pi_{2 k+1}\left(I_{0}\right)=K_{k+1}$. Let $J_{k+1}:=\pi_{2 k+1}\left(I_{k+1}\right)$. Since $\pi_{2 k+1}$ is an isometry, $J_{k+1} \cap K_{i}=\emptyset$ for all $i \in\{1, \ldots, k+1\}$.

In the even step, we first define the isometry $\sigma_{2 k+2}: J_{k+1} \rightarrow K_{k+1}$ by the condition $\sigma_{2 k+2}\left(\pi_{2 k+1}(t)\right)=\pi(t)$ for every $t \in I_{k+1}$. Next, the isometry $\pi_{2 k+2}$ of $[0, \Delta+1+\varepsilon]$ is defined by

$$
\pi_{2 k+2}(t):= \begin{cases}\sigma_{2 k+2}\left(\pi_{2 k+1}(t)\right) & \text { if } t \in I_{k+1}, \\ \sigma_{2 k+2}^{-1}\left(\pi_{2 k+1}(t)\right) & \text { if } t \in I_{0} \\ \pi_{2 k+1}(t) & \text { otherwise. }\end{cases}
$$

It follows directly from the definition of $\pi_{2 k+2}$ that

$$
\forall i \in\{1, \ldots, k+1\}, \quad \forall t \in I_{i}, \quad \pi_{2 k+2}(t)=\pi(t) .
$$

Notice that $\pi_{2 k+2}\left(I_{0}\right)=J_{k+1}$.

When $k=s-1$, the set $K_{k+1}=K_{s}$ can have measure smaller than $\delta$. We partition $J_{s-1}:=\pi_{2 s-2}\left(I_{0}\right)$ into two sets $J_{s-1}^{\prime}$ and $J_{s-1}^{\prime \prime}$ such that $\mu\left(J_{s-1}^{\prime}\right)=\mu\left(K_{s}\right)$. We then continue in the same manner and define an isometry $\pi_{2 s-1}$ that agrees with $\pi$ on all the sets $I_{i}$ with $i \in\{1, \ldots, s-1\}$ and $\pi_{2 s-1}\left(I_{0}\right)=K_{s} \cup J_{s-1}^{\prime \prime}$. Finally, we define an isometry $\pi_{2 s}$ that agrees with $\pi$ on $\left[0, \Delta+1+\varepsilon^{\prime}\right]$.

Let us now define a coloring $c: V(T) \cup E(T) \rightarrow 2^{[0, \Delta+1+\varepsilon]}$ as follows:

$$
c(x):= \begin{cases}\pi_{i}\left(c_{0}(x)\right) & \text { if } x \text { has level } i \in\{0, \ldots, 2 s\} \\ X & \text { if } x=r .\end{cases}
$$


Since $\pi_{i}$ is an isometry for $i \in\{0, \ldots, 2 s\}$ and $c_{0}$ is a fractional total coloring of $T$, we have $\mu(c(x)) \geq 1$ for all $x \in V(T) \cup E(T)$. To prove that $c$ is a fractional $(\Delta+1+\varepsilon)$-total coloring of $T$ it suffices to prove that $c(x) \cap c(y)=\emptyset$ if $x$ and $y$ are adjacent or incident in $T$. The levels of $x$ and $y$ can differ by at most 1 . If the levels of $x$ and $y$ are the same, then $c_{0}(x) \cap c_{0}(y)=\emptyset$ since $c_{0}$ is a fractional total coloring of $T$. Hence,

$$
c(x) \cap c(y)=\pi_{i}\left(c_{0}(x)\right) \cap \pi_{i}\left(c_{0}(y)\right)=\emptyset .
$$

Let $x$ be a vertex or an edge of level $i$ and let $y$ be a vertex or an edge adjacent to $x$ of level $i+1$, with $i \in\{0, \ldots, 2 s-1\}$. Since $c_{0}(x) \cap c_{0}(y)=\emptyset$, we have $\pi_{i}\left(c_{0}(x)\right) \cap \pi_{i}\left(c_{0}(y)\right)=\emptyset$ as well. Since $c_{0}$ uses only colors from the interval $\left[0, \Delta+1+\varepsilon^{\prime}\right]$, we also have $\pi_{i}\left(c_{0}(x)\right) \cap \pi_{i}\left(I_{0}\right)=\emptyset$. The isometry $\sigma_{i}$ interchanges (some of the) colors from $\pi_{i}\left(I_{0}\right)$ (not used in the level $i$ ) with some of the other colors, and hence, $c(y)=\pi_{i+1}\left(c_{0}(y)\right) \subseteq \pi_{i}\left(c_{0}(y)\right) \cup \pi_{i}\left(I_{0}\right)$. Therefore,

$$
c(x) \cap c(y) \subseteq\left(\pi_{i}\left(c_{0}(x)\right) \cap \pi_{i}\left(c_{0}(y)\right)\right) \cup\left(\pi_{i}\left(c_{0}(x)\right) \cap \pi_{i}\left(I_{0}\right)\right)=\emptyset .
$$

If $x$ has level $2 s$ and $y$ has level $2 s+1$, then $y=r$ and $c(y)=X=\pi\left(c_{0}(r)\right)$. On the other hand, $c(x)=\pi_{2 s}\left(c_{0}(x)\right)=\pi\left(c_{0}(x)\right)$. Therefore, the sets are disjoint. To conclude, notice that $c(x)=c_{0}(x)$ for all vertices and edges of level 0 and that $c\left(r r^{\prime}\right)=\pi_{2 s}\left(c_{0}\left(r r^{\prime}\right)\right)=\pi\left(c_{0}\left(r r^{\prime}\right)\right)=Y$.

We are now ready to prove Theorem 6 ,

Proof of Theorem [6. Fix $\varepsilon^{\prime} \in(0, \varepsilon)$. Let $d$ be large enough so that Lemma 13 holds for the values of $\Delta, \varepsilon^{\prime}$ and $\varepsilon$. Set $d_{0}:=2 d+2$ and let $g$ be greater than $\left(d_{0}+1\right) \cdot \Delta$ and such that Lemma 12 holds for $\Delta$ and $\varepsilon^{\prime}$.

We proceed by induction on $|E(G)|$, the conclusion being trivial when $|E(G)| \leq \Delta$. Now, if $G$ is cyclically $\Delta$-edge-connected, then Lemma 12 yields the result (since $\varepsilon>\varepsilon^{\prime}$ ). So, we assume that $G$ is not cyclically $\Delta$ edge-connected. Let $F$ be a (minimal) cyclic edge-cut of $G$ such that $|F|<\Delta$ and $G-F$ is composed of two connected components $A$ and $B$ such that $|B|$ is minimized.

Let $F^{\prime}$ be the $d_{0}$-connector of $F$ in the subgraph $G_{X}$ of $G$ induced by $B \cup F$. We now show that the subgraph $G_{A}$ of $G$ induced by $A \cup N\left(F^{\prime}\right)$ has less edges than $G$. By Lemma 8 , we know that $N\left(F^{\prime}\right)$ is a forest. On the other hand, $B$ contains a cycle and hence $\left|E\left(N\left(F^{\prime}\right)\right) \backslash F\right|<|E(B)|$ (since $N\left(F^{\prime}\right)$ is contained in $G_{X}$ and the subgraph induced by $F$ is acyclic by the girth requirement). Hence, $G_{A}$ has less edges than $G$, maximum degree at 
most $\Delta$ and girth at least $g$. Therefore, there exists a fractional $(\Delta+1+\varepsilon)$ total coloring $c_{A}$ of $G_{A}$ : if the maximum degree of $G_{A}$ is $\Delta$, then this follows from the induction hypothesis, and otherwise it follows from Theorem 3 ,

Let $G_{B}$ be the graph obtained from $G$ by contracting $A$ into a single vertex $w$, and then subdividing $\lfloor g / 2\rfloor$ times each edge incident with $w$; thus the distance between $w$ and $B$ in $G_{B}$ is greater than $\lfloor g / 2\rfloor$. Hence, the girth of $G_{B}$ is at least $g$. Since $G_{B}$ contains more than $\Delta$ edges, $G_{B}$ is cyclically $\Delta$-edge-connected, because any cyclic edge-cut of $G_{B}$ yields a cyclic edgecut of $G$ of at most the same order, and whose removal splits $G$ into two components one of which is smaller than $B$. Consequently, Lemma 12 ensures the existence of a fractional $\left(\Delta+1+\varepsilon^{\prime}\right)$-total coloring $c_{B}$ of $G_{B}$.

Let $\mathscr{E}$ be the set of edges $x y$ of $G$ with $x \in V\left(F^{\prime}\right)$ and $y \in V(B) \backslash V\left(F^{\prime}\right)$, i.e., $e$ is in $N\left(F^{\prime}\right)$ but not in $F^{\prime}$. For every $e=x y \in \mathscr{E}$ with $x \in V\left(F^{\prime}\right)$, let $T_{0}(y)$ be the subgraph of $G_{X}-V\left(F^{\prime}\right)$ induced by the vertices at distance at most $d$ from $y$. Let $T(e)$ be the graph obtained from $T_{0}(y)$ by adding $x$ and the edge $e=x y$. Observe that $T(e)$ is a tree, because the girth $g$ of $G$ is greater than $2 d+2$. Moreover, if $e^{\prime}=x^{\prime} y^{\prime} \in \mathscr{E}$ is distinct from $e$ (and $\left.x^{\prime} \in V\left(F^{\prime}\right)\right)$, then $T(e)$ and $T\left(e^{\prime}\right)$ are vertex-disjoint unless $x=x^{\prime}$ and then $x$ is the unique common vertex of $T(e)$ and $T\left(e^{\prime}\right)$, because $F^{\prime}$ is $d_{0^{-}}$-closed.

Now, for every edge $e=x y \in \mathscr{E}$, we apply Lemma 13 to the tree $T(e)$ with $c_{0}:=c_{B}, r:=x, X:=c_{A}(x)$ and $Y:=c_{A}(e)$. This yields a fractional $(\Delta+1+\varepsilon)$-total coloring $c_{e}$ of $T(e)$, which agrees with $c_{B}$ on all the leaves and edges incident to a leaf that are at distance $d$ from $x$, if any.

The property of disjointness of the trees ensures that $c_{B}$ along with all the colorings $c_{e}$ for $e \in \mathscr{E}$ yield a fractional $(\Delta+1+\varepsilon)$-total coloring $c^{\prime}$ of the subgraph $B^{\prime}$ of $G$ spanned by $B-F^{\prime}$ and $\mathscr{E}$.

Since $G_{A} \cup B^{\prime}=G$ and the colorings $c_{A}$ and $c^{\prime}$ agree on all the edges in $\mathscr{E}$ and all the vertices of $F^{\prime}$ that are incident to an edge in $\mathscr{E}$, we obtain a fractional $(\Delta+1+\varepsilon)$-total coloring of $G$, as wanted.

\section{References}

[1] M. Behzad. Graphs and their chromatic numbers. Doctoral thesis, Michigan State University, 1965.

[2] T. Ito, W. S. Kennedy, and B. Reed. A characterization of graphs with fractional total chromatic number equal to $\Delta+2$. In Proceedings of the $V$ Latin-American Algorithms, Graphs and Optimization Symposium (LAGOS 2009), Electronic Notes in Discrete Math. To appear. 
[3] T. Kaiser, A. King, and D. Král'. Fractional total colourings of graphs of high girth, 2009. Manuscript arXiv:0911.2808.

[4] T. Kaiser, D. Král', R. Škrekovski, and X. Zhu. The circular chromatic index of graphs of high girth. J. Combin. Theory Ser. B, 97(1):1-13, 2007.

[5] K. Kilakos and B. Reed. Fractionally colouring total graphs. Combinatorica, 13(4):435-440, 1993.

[6] L. Lovász. Combinatorial problems and exercises. AMS Chelsea Publishing, Providence, RI, second edition, 2007.

[7] M. Molloy and B. Reed. A bound on the total chromatic number. Combinatorica, 18(2):241-280, 1998.

[8] B. Reed. Fractional total colouring. Presentation at the DIMACS Workshop on Graph Coloring and Structure, Princeton, May 2009.

[9] E. R. Scheinerman and D. H. Ullman. Fractional graph theory. WileyInterscience Series in Discrete Mathematics and Optimization. John Wiley \& Sons Inc., New York, 1997.

[10] V. G. Vizing. Some unsolved problems in graph theory. Uspehi Mat. Nauk, 23(6 (144)):117-134, 1968.

[11] H. P. Yap. Total colourings of graphs, volume 1623 of Lecture Notes in Mathematics. Springer-Verlag, Berlin, 1996. 